\title{
DESCRIÇÃO DA DIVERSIDADE GENÉTICA DE POPULAÇÕES NATURAIS DE BARBATIMÃO Stryphnodendron adstringens (Mart.) Coville EM UNIDADES DE CONSERVAÇÃO DE MINAS GERAIS ${ }^{1}$
}

\author{
Jaqueline Siqueira Glasenapp², Vicente Wagner Dias Casali², Ernane Ronie Martins³, Cosme Damião \\ Cruz $^{4}$ e Priscila Barros Barbosa ${ }^{2}$
}

\begin{abstract}
RESUMO - O objetivo deste estudo foi descrever a diversidade genética de $S$. adstringens em três populações localizadas em Unidades de Conservação do Estado de Minas Gerais, utilizando-se marcadores isoenzimáticos. Foram amostradas as populações dos Parques Estaduais (PE) do Biribiri e Serra Nova e Parque Nacional (PN) das Sempre Vivas. Foram empregados 14 marcadores isoenzimáticos, dos quais nove locos foram polimórficos (est-2, est-3, est-4, got-1, pgi-1, $m d h-4$, idh-1, $a d h-1$ e $s k d h-1)$. Os testes de ajustamento às proporções genotípicas de EHW, teste exato de Fisher e $\chi^{2}$ não foram significativos nos locos isoenzimáticos das três populações, exceto est-4 no PE Serra Nova, $m d h-4$ e idh-1 no PE do Biribiri. Os valores de heterozigosidade média esperada $\left(\mathrm{H}_{e}\right)$ variaram entre 0,217 e 0,255, estando próximos dos encontrados em espécies arbóreas tropicais $(0,204$ e 0,211) com as mesmas características de distribuição geográfica de $S$. adstringes. O índice de fixação $(F)$ foi significativamente menor do que zero na população do PE do Biribiri $(-0,149)$ e não significativo no PN das Sempre Vivas $(-0,031)$ e no PE Serra Nova $(-0,138)$. O excesso significativo de heterozigotos estimado na população do PE do Biribiri pode significar seleção a favor desses genótipos, porém, para verificar tal possibilidade, são requeridos estudos que consideram variáveis ambientais.
\end{abstract}

Palavras-chave: Diversidade genética; Cerrado; Plantas medicinais.

\section{DESCRIPTION OF GENETIC DIVERSITY OF NATURAL POPULATIONS OF BARBATIMÃO Stryphnodendron adstringens (Mart.) Coville IN CONSERVATION AREAS FROM MINAS GERAIS}

\begin{abstract}
The aim of this study was to describe the genetic diversity of $\mathbf{S}$. adstringens in three populations from protected areas of Minas Gerais State, Brazil, by using isozymes. We sampled populations from State Parks (SP) Biribiri and Serra Nova, and National Park (NP) Sempre Vivas. We used 14 isozyme markers, which among them nine loci were polymorphic (est-2, est-3, est-4, got-1, pgi-1, mdh-4, idh-1, adh-1 and skdh-1). The adjustment tests to EHW genotypic proportions, Fisher's exact test and $\chi^{2}$, were not significant to the isozyme loci in the three populations, except est-4 in SP Serra Nova, mdh-4 and idh-1 in SP Biribiri. The values of average expected heterozygosity $\left(H_{e}\right)$ ranged between 0.217 to 0.255 , and are close to those found in tropical tree species (0.204 to 0.211) with the same characteristics of geographical distribution of S. adstringes. The fixation index (F) were significantly lower than zero in the population of SP Biribiri (-0.149), and not significantly in the NP Sempre Vivas (-0.031) and SP Serra Nova (-0.138). The significant excess of heterozygotes in SP Biribiri population may be an indicative of selection in favor of these genotypes, however, to verify this possibility, further studies are required, considering environmental variables.
\end{abstract}

Keywords: Genetic diversity; Savannah; Medicinal plants.

\footnotetext{
${ }^{1}$ Recebido em 25.02.2011 aceito para publicação em 07.11.2013.

22Departamento de Fitotecnia da Universidade Federal de Viçosa, UFV, Brasil. E-mail:<siqueiragaia@yahoo.com.br>, <vwcasali@ufv.br> e <psilinham@gmail.com>

${ }^{3}$ Instituto de Ciências Agrárias da Universidade Federal de Minas Gerais, UFMG, Brasil. E-mail:<ernane-martins@ufmg.br>

${ }^{4}$ Departamento de Biologia Geral da Universidade Federal de Viçosa, UFV, Brasil. E-mail:<cdcruz@ufv.br>
} 


\section{INTRODUÇÃO}

A espécie arbórea do Cerrado (Stryphnodendron adstringens), conhecida popularmente como "barbatimão”, tem ampla distribuição geográfica. Ocorre, principalmente, no Cerrado típico, campo-sujo e Cerradão, desde o Pará, Planalto Central, Minas Gerais até São Paulo (FELFILI et al., 1999). A casca do barbatimão é conhecida por suas propriedades medicinais e tanantes, as quais são devidas às altas concentrações de compostos fenólicos (PANIZZA et al., 1988; TEIXEIRAet al., 1990; MELLO et al., 1996). A madeira pode ser utilizada para diversos fins, pois é pesada, dura e resistente à ação da água e do sol (LORENZI, 1992). Atualmente, o desmatamento vem reduzindo drasticamente as áreas cobertas por Cerrado e, consequentemente, por $S$. adstringens (FELFILI; SILVA JÚNIOR, 2001; FELFILI et al., 2002). Além disso, a prática tradicional da extração da casca é feita sem nenhum critério, muitas vezes levando os indivíduos à morte (FELFILI; BORGES FILHO, 2004).

Os estudos com espécies arbóreas nativas iniciaram-se, principalmente, a partir do final da década de 1980, quando alguns centros de pesquisa passaram a dar atenção à conservação dos recursos genéticos (FREITAS et al., 2005). A avaliação da diversidade genética é considerada vital para a formulação de estratégias de conservação para espécies ameaçadas de extinção; entre as espécies ameaçadas, encontram-se as plantas medicinais (SEBATIAN et al., 2010). Somente com a conservação da diversidade genética podem ser mantidos os processos evolutivos atuantes nas populações. A utilização de técnicas isoenzimáticas poderia aumentar grandemente o entendimento sobre a organização genética dessas espécies. Como dados eletroforéticos podem ser obtidos a partir de praticamente qualquer espécie e não requerem conhecimento prévio do genoma, esses marcadores são adequados para a caracterização da estrutura genética de populações naturais de plantas tropicais.

Diante da necessidade do estabelecimento de políticas voltadas ao manejo e conservação de S. adstringens, este trabalho teve como objetivo descrever a diversidade genética de três populações localizadas em Unidades de Conservação do Estado de Minas Gerais, utilizando marcadores isoenzimáticos. Essas informações associadas a outras informações como ecologia, biologia reprodutiva, modo de dispersão de sementes e componentes da historia vital podem ser utilizadas de maneira a assegurar os processos evolutivos atuantes, seja na conservação, seja no manejo de populações naturais.

\section{MATERIAL E MÉTODOS}

\subsection{Amostragem}

As coletas foram realizadas em Unidades de Conservação de Minas Gerais no mês de outubro de 2009. Foram amostradas as populações dos Parques Estaduais (PE) do Biribiri (altitude de $1.173 \mathrm{~m}$ ) e Serra Nova (altitude de 963 m) e do Parque Nacional (PN) das Sempre Vivas (altitude de 1.305 m). O PE do Biribiri, coordenadas geográficas S 18¹2.48.1”' - W 43³7’04.5”, e PN das Sempre vivas (S 1754'47.5”' - W 4348'08.6”') localizam-se na Mesorregião do Jequitinhonha (IBGE, 1997), no Município de Diamantina, MG. Essa região possui solos rasos, arenosos, pedregosos, ácidos e clima ameno. A vegetação encontra-se bem conservada, provavelmente devido ao fato de os solos não serem propícios à agropecuária. O PE Serra Nova (S 15³8'51.3”, - W 4243'09.4”) localiza-se na Mesorregião Norte mineira (IBGE, 1997), próximo ao Rio Pardo de Minas. O clima é semiárido, com temperaturas elevadas, e a vegetação encontra-se relativamente bem conservada, inclusive no entorno do Parque, sendo observadas extensas áreas com vegetação nativa. O número de indivíduos amostrados foi 34 em Serra Nova, 34 em Sempre Vivas e 37 em Biribiri. Foram coletados aproximadamente cinco frutos por indivíduo, observando-se a distância mínima de 50 m entre indivíduos dentro de populações (GONÇALVES et al., 2010).

\subsection{Extração enzimática e eletroforese}

As análises do polimorfismo aloenzimático foram realizadas no Laboratório de Melhoramento de Hortaliças da UFV, por meio da técnica de eletroforese em gel de amido. Sementes dos indivíduos amostrados foram incubadas em câmara de crescimento por três dias. Das plântulas germinadas, apenas uma oriunda de cada um dos indivíduos amostrados, em cada população, foi utilizada na extração de isoenzimas e nas análises genéticas. Na extração isoenzimática, as plântulas foram maceradas em almofariz, e a proporção de $1 \mathrm{~g}$ do macerado de cada plântula foi adicionado a cada $3 \mathrm{~mL}$ da solução extratora $n^{\circ} 1$, recomendada por Alfenas et al. (2006). Os géis foram preparados com $12 \mathrm{~g}$ de 
sacarose e 60 g de amido em 500 mL de solução-tampão do gel. Os seguintes sistemas-tampão foram utilizados: I) Tampão-eletrodo composto por 4,0 g. $\mathrm{L}^{-1} \mathrm{NaOH}$, 18,55 g.L.-1 ácido bórico, pH 7,9; tampão-gel composto por $40 \mathrm{~mL} . \mathrm{L}^{-1}$ solução do eletrodo, 1,84 g.L -1 $^{-1}$ Tris, 0 ,69 g.L ${ }^{-1}$ ácido cítrico, pH 8,6 (SOLTIS et al., 1983). II) Tampãoeletrodo composto por 16,35 g.L ${ }^{-1}$ Tris, 9,04 g.L.-1 ácido cítrico, pH 7,0; tampão-gel composto por 66,7 mL.L ${ }^{-1}$ da solução do eletrodo, pH 7,0, (SHAW; PRASAD, 1970). A pré-corrida foi conduzida a $15 \mathrm{~mA}$, tendo duração de 30 min com o uso do sistema-tampão I e 1 h com o uso do sistema-tampão II. A corrida foi realizada a 35 mA e durou cerca de 5 h. Os sistemas aloenzimáticos analisados com sistema-tampão I foram: esterase (EC 3.1.1.1), fosfatase ácida (EC 3.1.3.2), fosfoglucomutase (EC 5.4.2.2), fosfogluco isomerase (EC 5.3.1.9), glucose desidrogenase (EC 1.1.1.47), glucose-6-fosfato desidrogenase (EC 1.1.1.49), glutamato oxaloacetato transaminase (EC 2.6.1.1), leucina aminopeptidase (EC 3.4.11.1), peroxidase (EC 1.11.1.7) e glicerato-2desidrogenase (EC 1.1.1.29). Os sistemas isoenzimáticos analisados com o sistema-tampão II foram: álcool desidrogenase (EC 1.1.1.1), isocitrato desidrogenase (EC 1.1.1.42), malatodesidrogenase (EC 1.1.1.37) e xiquimato desidrogenase (EC 1.1.1.25)

\subsection{Análise dos dados eletroforéticos}

Nos géis pertinentes a cada sistema isoenzimático, foram identificadas regiões de atividade, locos e alelos. Os locos isoenzimáticos foram definidos com as mesmas abreviações utilizadas na designação de cada enzima (ex. MDH), porém com letras minúsculas e itálicas, seguidas por ordenação numérica crescente a partir do loco de migração mais lenta (ex. mdh-1). Os alelos em cada loco foram ordenados alfabeticamente, sendo o alelo de migração mais rápida identificado com a letra $a$ e os de migração mais lenta, seguindo a ordem.

Os testes de ajuste das proporções genotípicas observadas às esperadas pelo modelo de equilíbrio de Hardy-Weinber foram realizados, utilizando-se os testes $X^{2}$ e exato de Fisher. Um dos mais robustos testes de EHW é o teste $X^{2}$, porém a sua correta utilização requer que todos os valores esperados sejam $\geq 1$ e que não menos do que $20 \%$ sejam $\leq 5$ (COCHRAN, 1954). O teste de Fisher tem como vantagem o fato de não ser afetado por pequenos valores esperados, sendo apropriado a pequenos tamanhos amostrais e alelos com frequências muito baixas (LESSIOS, 1992).
Os desvios do EHW também foram inferidos pela estimativa do índice de fixação $F$ (WRIGHT, 1951). Para verificar se os valores de $F$ eram significativamente diferentes de zero dentro de populações, foi utilizado o método proposto por Li e Horvitz (1953). Estes pesquisadores reformularam o teste $X^{2}$ em termos da estatística $F$ como $x^{2}=F^{2} \mathrm{~N}(\mathrm{a}-1)$, com graus de liberdade $\mathrm{df}=\frac{\mathrm{a}(\mathrm{a}-1)}{2}$ em que $N$ é igual ao número de indivíduos amostrados e $a$ é o número de alelos. O F é calculado usando-se a expressão $\mathrm{F}=1-\frac{\mathrm{H}_{0}}{\mathrm{H}_{\mathrm{e}}}$, em que a heterozigosidade esperada é dada por $\mathrm{H}=1-\sum \mathrm{p}_{\mathrm{i}}^{2}$ e $p_{i}$ é a frequência do i-ésimo alelo na população (NEI, 1973). Cada loco em cada população foi testado separadamente, e os valores de $X^{2}$ e os graus de liberdade, somados sobre todos os locos, obtendo-se, dessa forma, o valor total de $\chi$ do coeficiente médio $F$. No processamento dos dados, foi utilizado o aplicativo computacional em genética e estatística GENES (versão 1.0.0).

\section{RESULTADOS}

\subsection{Descrição da diversidade aloenzimática}

Dos 14 sistemas isoenzimáticos analisados, somente os sistemas EST, PGI, IDH, GOT, MDH, ADH e SKDH foram polimórficos e incluídos nas análises estatísticas. O sistema peroxidase (PER), apesar de polimórfico, não foi considerado devido à má resolução do padrão de bandas nos géis. Nos géis malatodesidrogenase $(\mathrm{MDH})$, foram evidentes três regiões de atividade. As regiões catodal e anodal parecem ser controladas por um loco cada, $m d h-1$ e $m d h-5$, respectivamente. A região intermediária é aparentemente constituída por dois locos fixados ( $m d h-2$ e $m d h-3)$ e um polimórfico ( $m d h-4)$. O loco $m d h-3$ se sobrepõe à banda híbrida do $m d h-4$ e, logo acima do loco $m d h$-2, são evidentes duas bandas que parecem ser o resultado da interação entre $m d h$ 2 e os alelos $a$ de $m d h-3$ e $b$ de $m d h-4$. Assim, devido à complexidade do padrão de bandas na região intermediária, somente o loco $m d h-4$, onde foram observados três alelos e o padrão característico de enzimas diméricas foi considerado nas análises estatísticas (Figura 1). Os locos $m d h-1$ e $m d h-5$ não foram suficientemente resolvidos a ponto de serem incluídos nas análises genéticas.

Revista Árvore, Viçosa-MG, v.38, n.1, p.103-112, 2014 


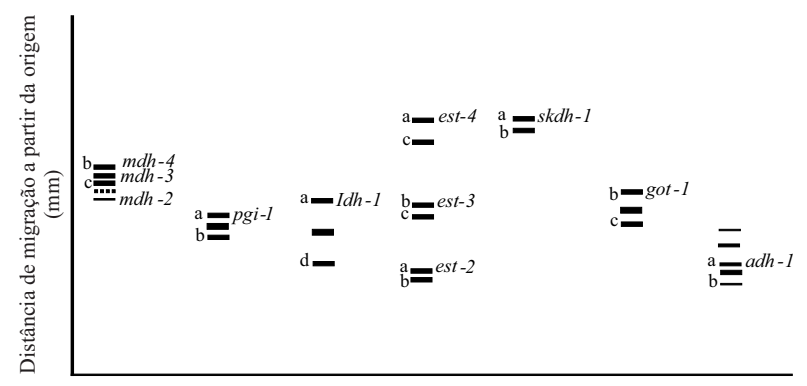

Figura 1 - Representação diagramática dos padrões de bandas dos locos isoenzimáticos polimórficos observados nas populações de Stryphnodendron adstringens. Letras minúsculas representam alelos que compõem locos (itálico).

Figure 1 - Diagrammatic representation of band patterns of isoenzymatic polymorphism observed in Stryphnodendron adstringens populations. Small letters represent alleles within loci (italic).

Nos géis fosfoglucoisomerase (PGI) foram observadas duas regiões de atividade controladas por um loco cada. No loco pgi-1 foi evidente o padrão de bandas dimérico e dois alelos (Figura 1). No loco pgi-2, apesar de aparentemente fixado, foi verificada pequena variação na distância de migração das bandas. Em isocitrato desidrogenase (IDH), foi observado um único loco (idh-1), quatro alelos e padrão de bandas dimérico (Figura 1). Nos géis esterases (EST), foram aparentes três regiões de atividade. Em cada uma das regiões anodal e intermediária, foi observado padrão monomérico com um loco e três alelos (est-3 e est -4). $\mathrm{Na}$ região catodal, foram verificados dois locos, est-2 com dois alelos (Figura 1) e est -1 fixado.

Em xiquimato desidrogenase (SKDH), foi evidente a estrutura quaternária monomérica, com uma única região de atividade representada por um loco e dois alelos (Figura 1). Nos géis glutamato oxaloacetato (GOT), foram observadas duas regiões de atividade. Na região anodal foram evidentes dois locos fixados (got-2 e got-3) e na catodal, o padrão de bandas dimérica com um loco (got-1) e três alelos (Figura 1). Uma única região de atividade com padrão de bandas triplex, que variaram em espessura e intensidade de cor, foi aparente em álcool desidrogenase (ADH). A banda de migração mais lenta foi mais espessa e corada que as demais, na maioria dos indivíduos, sendo menos corada em indivíduos que apresentaram padrão de bandas heterozigoto em relação a essa banda (adh-1). Assim, somente o loco (adh-1), onde foram observados estrutura dimérica e dois alelos, foi considerado nas análises estatísticas (Figura 1).

\subsection{Medidas de diversidade genética}

Resultados dos testes de ajustamento às proporções de EHW pelo teste exato de Fisher e teste de $X^{2}$ não diferiram significativamente em todos os locos isoenzimáticos, nas três populações, exceto est-4 no PE Serra Nova e $m d h-3$ e idh-1 no PE do Biribiri. Os locos est-4 no PE do Biribiri e skdh-1 no PN das Sempre Vivas também foram significativos, mas somente nas estimativas $X^{2}$ (Tabela 1).

Na população do PE Serra Nova, os locos got-1, $a d h-1$ e $s k d h-1$ foram fixados, bem como verificada a deficiência de heterozigotos no loco $m d h-3(0,204)$, sendo a heterozigosidade média esperada $\left(H_{e}\right) 0,231$. O loco est-1 foi fixado nas três populações. Na população do PN das Sempre Vivas, foi verificada substancial deficiência de heterozigotos nos locos skdh-1 $(0,469)$, sendo menos acentuada no loco got-1 $(0,269)$; a heterozigosidade média $\left(H_{e}\right)$ foi 0,217 e os locos est-2, pgi-1 e $a d h$-1, fixados. Na população do P. E. do Biribiri, foi verificada a deficiência de heterozigotos nos locos est-3 $(0,017), m d h-3(0,213)$ e $a d h-1(0,274)$, sendo a heterozigosidade média $\left(H_{e}\right)$ 0,255 e o loco pgi-1 fixado.

Os valores de heterozigosidade média esperada $\left(H_{e}\right)$ estimados dentro de populações variaram entre 0,217 e 0,255. Os resultados dos testes de significância do índice de fixação médio $F$, considerando todos os locos dentro de populações, não diferiram significativamente no PN das Sempre Vivas (-0,031) e no PE Serra Nova $(-0,138)$, com probabilidades iguais a 0,558 e 0,789, respectivamente. O resultado do índice de fixação na população do PE do Biribiri foi significativo $(-0,149)$, com probabilidade igual a 0,012 , indicando excesso de heterozigotos, que foi devido, essencialmente, aos locos est-4 ( $F=-0,446)$ e idh-1 $(F=-0,358)$. Entretanto, em locos $m d h-3(F=0,213)$ e $a d h-1(F=0,274)$ foi constatada substancial deficiência de heterozigotos. As frequências alélicas, heterozigosidade esperada $\left(H_{e}\right)$, heterozigosidade observada $\left(H_{o}\right)$ e índice de fixação $(F)$ nas populações estão descritos na Tabela 2.

\section{DISCUSSÃO E CONCLUSÃO}

O complexo-padrão de bandas observadas na região intermediária dos géis MDH possivelmente é devido à dimerização interlocos. Enzimas controladas por mais 
Tabela 1 - Teste $\chi^{2}$ e exato de Fisher de ajustamento das proporções genotípicas observadas e em S. adstringens, nas proporções esperadas sob EHW.

Table 1 - Fisher's exact and $\chi^{2}$ tests for the adjustment of the genotypes observed in Stryphnodendron adstringens to the proportions expected under EHW.

\begin{tabular}{|c|c|c|c|c|c|c|}
\hline \multirow[t]{2}{*}{ Loco } & \multicolumn{2}{|c|}{ P.E. Serra Nova } & \multicolumn{2}{|c|}{ PN Sempre Vivas } & \multicolumn{2}{|c|}{ P.E. do Biribiri } \\
\hline & $\mathrm{P}(\mathrm{A})$ & $\left(\chi^{2}\right)$ & $\mathrm{P}(\mathrm{A})$ & $\left(\chi^{2}\right)$ & $\mathrm{P}(\mathrm{A})$ & $\left(\chi^{2}\right)$ \\
\hline est-1 & 1,0 & 1,0 & 1,0 & 1,0 & 1,0 & 1,0 \\
\hline est-2 & 1,0 & 0,931 & 1,0 & 1,0 & 1,0 & 0,525 \\
\hline est-3 & 0,728 & 0,461 & 0,258 & 0,259 & 1,0 & 0,682 \\
\hline est-4 & $0,037 *$ & $0,016 *$ & 0,082 & 0,051 & 0,0738 & $0,038 *$ \\
\hline got-1 & 1,0 & 0,788 & 0,214 & 0,117 & 1,0 & 0,933 \\
\hline pgi-1 & 0,557 & 0,211 & 1,0 & 1,0 & 1,0 & 1,0 \\
\hline mdh-3 & 0,290 & 0,235 & 1,0 & 0,573 & $0,000 *$ & $0,000 *$ \\
\hline idh-1 & 1,0 & 0,963 & 0,728 & 0,461 & $0,001 *$ & 0,001 * \\
\hline adh-1 & 1,0 & 1,0 & 1,0 & 1,0 & 0,197 & 0,095 \\
\hline skdh-1 & 1,0 & 1,0 & 0,089 & $0,007 *$ & 1,0 & 0,866 \\
\hline
\end{tabular}

$\mathrm{P}(\mathrm{A})=$ probabilidade acumulada do teste exato de Fisher. Níveis de significância: * significativon a 5\%. P(A): accumulated probability of Fisher's exact test. Levels of significance: * significant at $5 \%$.

de um loco gênico podem formar moléculas híbridas entre locos, coincidindo, às vezes, com outras quanto à posição no gel (ALFENAS; BRUNE., 1998). Além do mais, a dimerização entre locos MDH tem sido bem observada em algumas espécies (GOODMAN et al., 1980). Assim, as duas bandas observadas logo acima do loco $m d h-2$ podem ser devidas à interação desse loco com os locos mdh-3 e $m d h-4$ ou a modificações posteriores na estrutura quaternária dos produtos dos alelos desses locos ( $m d h-3$ e $m d h-4)$. Modificações pós-transcricionais podem resultar em bandas secundárias ou rastros corados no gel (ALFENAS et al., 1998). Foi observada a estrutura quaternária dimérica nas isoenzimas $\mathrm{MDH}$ em $S$. adstringens. A estrutura dimérica para as isoenzimas MDH foi igualmente observada em diversos organismos incluindo archaea, eubacteria, fungi, plantas e mamíferos (MUSRATI, 1998). Brune et al. (2006) descreveram MDH como composta por duas ou quatro subunidades, em que, frequentemente, são observados dois ou mais locos envolvidos.

A estrutura quaternária dimérica constatada nas isoenzimas PGI em S. adstringens está de acordo com o observado em muitos outros organismos, como fungos, bactérias, plantas e animais, incluindo o ser humano (NOLTMANN, 1972; HIZUKURI et al., 1975; TEKAMPOLSON et al., 1988; CINI et al., 1988; SUN et al., 1990; BRUNE et al., 2006). Como em S. adstringens, duas regiões de atividade são comumente evidentes nos géis PGI de outras espécies de plantas (ADAMS; JOLY, 1980; SOUZA, 2000).
As estruturas quaternárias de isocitrato desidrogenase (IDH) e esterase (EST) observadas em S. adstringens seguem o padrão. Muito estudada em fungos e animais, a enzima IDH é normalmente dimérica ou oligomérica (BRUNE et al., 2006), sendo a estrutura dimérica confirmada em plantas como Spondias tuberosa (SOUZA, 2000), Cucumis sativus (WATANABE et al., 2007) e cerejeira (GRANGER et al., 1992). Esterase é um dos sistemas enzimáticos mais polimórficos em plantas (WEEDEN; WENDEL, 1990) e o mais estudado em arroz (ENDO; MORISHMA, 1983), sendo as variantes dessas enzimas encontradas em plantas, geralmente monoméricas ou diméricas (BRUNE et al., 2006). Apesar de pouco estudada, em algumas espécies foram verificadas estruturas quaternárias monoméricas e diméricas para a enzima xiquimato desidrogenase (SKDH) (CHAUDHURI; COGGINS, 1985; MACLEAN etal., 2000). Como mencionado em $S$. adstringens, foi observada estrutura monomérica.

Em ambas as isoenzimas glutamato oxaloacetato (GOT) e álcool desidrogenase (ADH), foi observada estrutura quaternária dimérica. Não houve dúvidaspadrão das isoenzimas GOT, o que tem sido reconhecido como dimérico em plantas (ADAMS; JOLY, 1980; KEPHART, 1990; GRANGER et al., 1992; YAGI et al., 1993). Entretanto, o padrão triplex observado nos géis álcool desidrogenase (ADH) não parece ser devido à associação de bandas híbridas em indivíduos heterozigotos. A intensidade de cor e a espessura das bandas formadas dependem da frequência em que são 
Tabela 2 - Frequências alélicas, heterozigosidade esperada $\left(H_{e}\right)$, heterozigosidade observada $\left(H_{o}\right)$ e índice de fixação $(F)$ nas populações.

Table 2 - Allele frequency, expected heterozygosity $\left(H_{e}\right)$, observed heterozygosity $\left(H_{o}\right)$ and fixation index $(f)$ in the populations.

\begin{tabular}{|c|c|c|c|c|c|c|c|}
\hline \multirow{2}{*}{ Loco } & \multicolumn{4}{|c|}{ Alelos } & \multirow{2}{*}{$\mathrm{H}_{\mathrm{e}}$} & \multirow{2}{*}{$\mathrm{H}_{o}$} & \multirow{2}{*}{$F$} \\
\hline & $\mathrm{a}$ & $\mathrm{b}$ & $\mathrm{C}$ & $\mathrm{d}$ & & & \\
\hline \multicolumn{8}{|c|}{ Serra Nova State Park } \\
\hline est-2 & 0,0147 & 0,9853 & & & 0,029 & 0,029 & $-0,015$ \\
\hline est-3 & 0,5441 & 0,2941 & 0,1618 & & 0,591 & 0,676 & $-0,144$ \\
\hline est-4 & 0,5 & 0,1765 & 0,3235 & & 0,614 & 0,794 & $-0,293$ \\
\hline got-1 & 0,0 & 1,0 & 0,0 & & 0,0 & 0,0 & 1,0 \\
\hline$p g i-1$ & 0,1765 & 0,8235 & & & 0,291 & 0,354 & $-0,214$ \\
\hline$m d h-3$ & 0,0 & 0,8971 & 0,1029 & & 0,185 & 0,147 & 0,204 \\
\hline$I d h-1$ & 0,0781 & 0,4375 & 0,4531 & 0,0313 & 0,596 & 0,625 & $-0,048$ \\
\hline$a d h-1$ & 1,0 & 0,0 & & & 0,0 & 0,0 & 1,0 \\
\hline$S k d h-1$ & 1,0 & 0,0 & & & 0,0 & 0,0 & 1,0 \\
\hline Média & & & & & 0,231 & 0,262 & $-0,138$ \\
\hline \multicolumn{8}{|c|}{ Sempre Vivas National Park } \\
\hline est-2 & 0,0 & 1,0 & & & 0,0 & 0,0 & 1,0 \\
\hline est-3 & 0,6765 & 0,2647 & 0,0588 & & 0,469 & 0,441 & 0,059 \\
\hline est-4 & 0,4118 & 0,2353 & 0,3529 & & 0,650 & 0,765 & $-0,175$ \\
\hline got-1 & 0,0 & 0,9118 & 0,0882 & & 0,161 & 0,118 & 0,268 \\
\hline pgi-1 & 0,0 & 1,0 & & & 0,0 & 0,0 & 1,0 \\
\hline$m d h-3$ & 0,0 & 0,9118 & 0,0882 & & 0,161 & 0,176 & $-0,097$ \\
\hline$I d h-1$ & 0,1765 & 0,2353 & 0,5441 & 0,0441 & 0,615 & 0,676 & $-0,099$ \\
\hline$a d h-1$ & 1,0 & 0,0 & & & 0,0 & 0,0 & 1,0 \\
\hline$S k d h-1$ & 0,0 & 0,9412 & 0,0588 & & 0,111 & 0,059 & 0,468 \\
\hline Média & & & & & 0,217 & 0,223 & $-0,031$ \\
\hline \multicolumn{8}{|c|}{ Biribiri State Park } \\
\hline est-2 & 0,0946 & 0,9054 & & & 0,171 & 0,189 & $-0,104$ \\
\hline est-3 & 0,6528 & 0,2222 & 0,125 & & 0,509 & 0,5 & 0,017 \\
\hline est-4 & 0,3649 & 0,1622 & 0,473 & & 0,617 & 0,892 & $-0,446$ \\
\hline got-1 & 0,0417 & 0,9583 & 0,0 & & 0,080 & 0,083 & $-0,043$ \\
\hline pgi-1 & 0,0 & 1,0 & & & 0,0 & 0,0 & 1,0 \\
\hline$m d h-3$ & 0,0811 & 0,7703 & 0,1486 & & 0,378 & 0,297 & 0,213 \\
\hline$I d h-1$ & 0,0946 & 0,3784 & 0,5 & 0,0270 & 0,597 & 0,811 & $-0,358$ \\
\hline$a d h-1$ & 0,9189 & 0,0811 & & & 0,149 & 0,108 & 0,274 \\
\hline$S k d h-1$ & 0,9730 & 0,0270 & & & 0,053 & 0,054 & $-0,028$ \\
\hline Média & & & & & 0,255 & 0,293 & $-0,149 *$ \\
\hline
\end{tabular}

* Significativo a $5 \%$ de probability. * Significant et $5 \%$ of probability.

encontradas as diferentes formas da enzima (ALFENAS et al., 1998). Em indivíduos heterozigotos, a coloração da banda intermediária em uma isoenzima dimérica é mais intensa e espessa, devido à sua maior concentração enzimática, o que não foi observado nos géis $\mathrm{ADH}$. A maior intensidade de cor observada com maior frequência na banda de migração mais lenta parece ser resultado de sobreposição alélica, sendo na direção catodal a essa banda evidente o padrão de bandas diméricas característico de indivíduos heterozigotos (Figura 1). De acordo com Brune et al. (2006), as aloenzimas ADH são diméricas ou tetraméricas. Também, foi observada estrutura quaternária dimérica em plantas como Vicia faba (LEBLOVA; EL AHMAD, 1989), Zea mays (LAI et al., 1982), Oryza sativa (TONG; LIN, 1988) e Glycine max (TIHANYI et al., 1989).

Os resultados não significativos do teste $\chi^{2}$ devem ser considerados com cautela, pois em testes de EHW, como em qualquer outro teste estatístico, a inabilidade em rejeitar a hipótese nula não necessariamente indica sua validade (erro do tipo II). É possível que as frequências genotípicas em uma população na qual não haja cruzamentos aleatórios sejam distribuídas de forma que mimetizem a distribuição multinomial (LI, 1988). 
Como também é possível que fatores que causem desvios a partir das expectativas de EHW conduzam às frequências genotípicas a direções opostas, sendo o resultado final não significativo(WORKMAN, 1969; CAVALLI-SFORZA; BODMER, 1971). Resultados significativos, no entanto, definitivamente indicam que uma ou mais das condições de EHW não são asseguradas (BERG; HAMRICK, 1997). Porém, em relação aos resultados significativos observados somente da estatística $\chi^{2}$, é importante ressaltar que na maioria dos locos analisados foram verificados um alelo relativamente comum e um ou mais alelos raros dentro de locos. Nas estimativas $\chi^{2}$, alelos raros podem gerar valores muito pequenos no denominador, os quais inflam o estimador, causando excessiva rejeição da hipótese nula de EHW (erro tipo I).

As estimativas de heterozigosidade média esperada $\left(H_{e}\right)$ dentro de populações foram próximas das encontradas no estudo de espécies arbóreas tropicais $(0,204$ e 0,211) (LOVELESS, 1992), com as mesmas características de distribuição de $S$. adstringes. Os valores de $H_{e}$ estimados indicaram que em $S$. adstringes, como em outras espécies tropicais, há ocorrência de um alelo comum e um ou mais alelos raros dentro de locos (LOVELESS, 1992). Entretanto, no estudo da diversidade genética de populações de Dimorphandra mollis, espécie nativa do Cerrado, a diversidade genética de locos isoenzimáticos foi consideravelmente alta $(H=0,463)$, superior ao esperado em árvores tropicais (GONÇALVES et al., 2010). Entretanto, valores de diversidade genética em $D$. mollis ( $H=0,174$ a 0,287$)$, obtidos por meio da técnica de RAPD (OLIVEIRA et al., 2008), foram mais próximos do observado em $S$. adstringes. Na avaliação da diversidade genética da coleção de germoplasma de Jatropha curcas utilizando marcadores SSR, a média estimada do índice de diversidade genética $\left(\mathrm{H}_{e}\right)$ foi relativamente alta $(0,5572)$, mostrando alto nível de diversidade da espécie (WEN, 2010). No estudo da diversidade e estrutura genética de duas populações de Myracrodruon urundeuva (aroeira), usando marcadores microssatélites, o maior valor de heterozigosidade esperado foi detectado na população de Selvíria, MS $\left(\mathrm{H}_{e}=0,749\right)$, enquanto Aramina, SP, teve $\mathrm{H}_{e}=0,678$ (VIEGAS et al., 2011).

Os resultados do índice de fixação dentro de populações em $S$. adstringens indicaram o excesso de heterozigotos mais acentuado $(-0,031$ a $-0,149)$ que o constatado em $D$. mollis ( $F=-0,018$ ) (GONÇALVES et al., 2010). Em outras espécies nativas do Cerrado como Euterpe edulis, Annona crassiflora e Eugenia dysenterica, os índices de fixação estimados dentro de populações foram positivos, indicando deficiência de heterozigotos (GAIOTTO et al., 2003; TELLES et al., 2003a,b).

Resultados não significativos do índice de fixação $(F)$ podem ser considerados como indicativos de cruzamentos aleatórios dentro das populações do PE Serra Nova e do PN Sempre Vivas. Contudo, valores significativos de $F$ como o observado no PE do Birbiri são frequentemente interpretados como seleção a favor de genótipos heterozigotos (RIBAS; KAGEYAMA, 2004; MELO JR. et al., 2004). Apesar de o polimorfismo isoenzimático ser neutro (KIMURA, 1968), há possibilidade de vantagem adaptativa de genótipos heterozigotos (KALISZ, 1989). Além do mais, os locos isoenzimáticos podem ser indiretamente selecionados quando ligados a QTLs relacionados a características adaptativas e apresentar frequências genotípicas em desacordo com o esperado (EHW). Entretanto, a verificação de tais possibilidades requereria estudos adicionais, no PE do Birbiri, como aqueles que consideram variáveis ambientais (LANDE, 1976, 1992; LYNCH; HILL, 1986; SPITZE, 1993; LYNCH, 1994).

\section{AGRADECIMENTOS}

Ao Conselho Nacional de Desenvolvimento Científico Tecnológico (CNPq), pela oportunidade dada à primeira autora de cursar o doutorado; à Fundação de Amparo à Pesquisa de Minas Gerais (FAPEMIG) e ao (CNPq), pelo apoio financeiro; e ao Instituto Estadual de Florestas (IEF) e, especialmente, aos diretores e funcionários das Unidades de Conservação e ao Instituto de Ciências Agrárias da UFMG, pelo apoio.

\section{REFERÊNCIAS}

ADAMS, W. T.; JOLY, E. J. Genetics of allozyme variants in loblolly pine. The Journal of Heredity, v.71, n.1, p.33-40, 1980.

ALFENAS, A. C.; BRUNE, W. Eletroforese em gel de amido. In: ALFENAS, A. C. (Ed.)

Eletroforese de aloenzimas e proteínas afins: fundamentos e aplicações em plantas e microorganismos. Viçosa, MG: Universidade Federal de Viçosa, 1998. p.60-63.

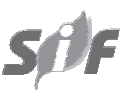

Revista Árvore, Viçosa-MG, v.38, n.1, p.103-112, 2014 
ALFENAS, A. C. et al. Eletroforese e marcadores bioquímicos em plantas e microorganismos. 2.ed. Viçosa, MG: Universidade Federal de Viçosa, 2006. 627p.

BERG, E. E.; HAMRICK, J. L. Quantification of genetic diversity at allozyme loci. Canadian Journal of Forest Research, v.27, n.1, p.215-424, 1997.

BRUNE, W., ALFENAS, A. C.; JUNGHANS, T. G. Identificações específicas de enzimas em géis. In: ALFENAS, A. C. (Ed.).

Eletroforese e marcadores

bioquímicos em plantas e

microrganismos Viçosa, MG: Universidade

Federal de Viçosa, 2006. p.202-328.

CAVALLI-SFORZA, L. L.; BODMER, W. F. The genetics of human populations. San Francisco: Freeman and Company,1971.

CHAUDHURI, S.; COGGINS, J. R. The purification of shikimate dehydrogenase from Escherichia coli. Biochemical Journal, v.226, p.217-223, 1985.

CINI, J. K.; COOK, P. F.; GRACY, R. W. Molecular basis for the isozymes of bovine glucose-6phosphate isomerase. Archives of

Biochemistry and Biophysics, v.263, n.1, p.96-106, 1988.

COCHRAN, W. G. Some methods for strengthening the common $\chi^{2}$ tests. Biometrics, v.10, p.417-451, 1954.

ENDO, T.; MORISHMA, H. Rice. In: TANKSLEY, S. D.; ORTTON, T. (Ed.) Isozymes in plant genetics and breeding. Amsterdam: Elsevier, 1983. Part B. p.129-146.

FELFILI, J. M. et al. Estudo fenológico de Stryphnodendron adstringens (Mart.) Coville no cerrado sensu stricto da Fazenda Água Limpa no Distrito Federal. Brasil. Revista Brasileira de Botânica, v.22, n.1, p.83-90, 1999.

FELFILI, J. M.; SILVA JÚNIOR, M. C. Diversidade Alfa e Beta no cerrado sensu stricto da Chapada Pratinha, Brasil. Acta Botânica Brasílica, v.15, n.2, p.243-254, 2001.
FELFILI, J. M. et al. Composição florística e fitossociológica do cerrado sentido restrito no município de Água Boa, MT. Acta Botânica Brasílica, v.16, n.1, p.103-112, 2002.

FELFILI, J. M.; BORGES FIFLHO, H. C. Extrativismo racional da casca de barbatimão [Stryphnodendron adstringens (Mart.) Coville]. Brasília: Universidade de Brasília, Departamento de Engenharia Florestal, 2004. 32p.

FREITAS, M. L. M. et al. Variabilidade genética intrapopulacional em Myracrodruon urundeuva Fr. All. por marcador AFLP. Scientia Forestalis, n.68, p.21-28, 2005.

GAIOTTO, F.A.; GRATTAPAGLIA, D.; VENCOVSKY, R. Genetic structure, mating system, and long-distance gene flow in Heart of Palm (Euterpe edulis Mar.). Journal of Heredity, v.94, n.5, p.399-406, 2003.

GONÇALVES, A. C. et al. Estrutura genética espacial em populações naturais de Dimorphandra mollis (Fabaceae) na região norte de Minas Gerais, Brasil. Revista Brasileira de Botânica, v.33, n.2, p.325-332, 2010.

GOODMAN, M. M. et al. Linkage relationships of 19 enzyme loci in maize. Genetics, v.96, p.697710,1980

GRANGER, A. R.; CLARKE, G. R.; JACSON, J. F. Sweet cherry identification by leaf isozyme polymorphism. Theoretical and Applied Genetics, v.86, p.458-464, 1993.

HIZUKURI, S.; TAKEDA, Y.; NIKUNI, Z. Glucose6-phosphate isomerase from peas. Methods in Enzymology, v.41, p.388-392, 1975.

INSTITUTO BRASILEIRO DE GEOGRAFIA E ESTATÍSTICA - IBGE. Diagnóstico ambiental da bacia do Rio Jequitinhonha: diretrizes gerais para a ordenação territorial. Salvador: 1997. $<$ www.ibge.gov.br>.

KIMURA, M. Evolutionary rate at the molecular level. Nature, v.217, p.624-626, 1968. 
KEPHART, S. R. Starch-gel electrophoresis of plant isozymes a comparative analisis of techniques. American Journal of Botany, v.77, p.693-712, 1990.

KALISZ, S. Fitness consequences of mating system, seed weight and emergence date in a winter annual, Collinsia verna. Evolution, v.43, p.1263-1272, 1989.

LAI, Y. K.; CHANDLEE, J. M.; SCANDALIOS, J. G. Purification and characterization of three nonallelic alcohol dehydrogenase isoenzymes in maize. Biochimica et Biophysica Acta, v.706, n.1, p.9-18, 1982.

LANDE, R. Natural selection and random genetic drift in phenotypic evolution. Evolution, v.30, p.314-334, 1976.

LANDE, R. Neutral theory of quantitative genetic variance in an island model with local extinction and colonization. Evolution, v.42, p.455-466, 1992.

LEBLOVA, S.; EL AHMAD, M. Characterization of alcohol dehydrogenase isolated from germinating bean (Vicia faba) seeds. Collection of Czechoslovak Chemical

Communications, v.54, p.2519-2527, 1989.

LESSIOS, H. A. Testing electrophoretic data for agreement with Hardy-Weinberg expectations. Marine Biology, v.112, p. 517-523, 1992

LI, C. C.; HORVITZ, D. G. Some methods of estimating the inbreeding coefficient. The American Journal of Human Genetics, v.5, n.1, p.107-117, 1953.

Li CC. Pseudo-random mating populations. In celebration of the 80th anniversary of the HardyWeinberg law. Genetics, v.119, p.731-737, 1988.

LORENZI, H. Árvores brasileiras: manual de identificação e cultivo de plantas arbóreas nativas do Brasil. Nova Odessa: Instituto Plantarum, 1992. v.1. 373p.

LOVELESS, M.D. Isozyme variation in tropical trees: patterns of genetic organization. New Forests, v.6, p.67-94, 1992.

LYNCH, M. Neutral models of phenotypic evolution. In: REAL, L.A. (Ed.) Ecological genetics Princeton: Princeton University Press, 1994. p.86-108.
LYNCH, M.; HILL, W. G. Phenotypic evolution by neutral mutation. Evolution, v.40, p.915-935, 1986.

MACLEAN, J. et al. Crystallization and preliminary X-ray analysis of shikimate dehydrogenase from Escherichia coli. Acta Crystallographica, v.56, p.512-515, 2000.

MELLO, J. C. P.; PETEREIT, F.; NAHRSTEDT, A. Flavan-3-ols and prodelphinidins from Stryphnodendron adstringens.

Phytochemistry, v.41, p.807-813, 1996.

MELO JUNIOR, A. F. et al. Estrutura genética de populações naturais de pequizeiro (Caryocar brasiliense Camb.). Scientia Forestalis, v.66, n.1, p.56-65, 2004.

MUSRATI, R. A. et al. Malate dehydrogenase: distribution, function and properties. General Physiology and Biophysics, v.17, n.3, p.193-210, 1998.

NEI, M. Analysis of gene diversity in subdivided populations. Proceedings of the National Academy of Sciences, v.70, p.3321-3323, 1973.

NOLTMANN, E. A. Aldose-ketose isomerases. In: BOYER, P. D. (Ed.) The enzymes. 3.ed. New York: Academic Press, 1972. v.6. p.271-354.

OLIVEIRA, D. A. et al. Variabilidade genética de populações de fava d'anta (Dimorphandra mollis) da região norte do Estado de Minas Gerais.

Revista Árvore, v.32, n.2, p.355-363, 2008.

PANIZZA, S. et al. Stryphnodendron barbadetiman (Velloszo) Martius: teor em tanino na casca e sua propriedade cicatrizante. Revista Brasileira de Ciências Farmacêuticas, v.10, n.1, p.101-106, 1988.

RIBAS, L. R.; KAGEYAMA, P. Y. Diversidade e estrutura genética em populações naturais de Trema micrantha (L.) B. Cientia Forestalis, n.66, p.66-75, 2004.

SEBASTIAN, V. A. et al. Assessment of Genetic Diversity within and among Populations of Tylophora rotundifolia Using RAPD Markers. Gene Conserve, v.9, v.37, p.94-117, 2010.

Revista Árvore, Viçosa-MG, v.38, n.1, p.103-112, 2014 
SHAW, C. R.; PRASAD, R. Starch gel electrophoresis of enzymes: a compilation of recipes. Biochemical Genetics, v.4, n.1, p.297-320, 1970.

SOLTIS, D. E. et al. Starch gel electrophoresis of fern: a compilation of grind buffers, gel and electrode buffers, and staining schedules. American Ferns Journal, v.73, n.1, p.9-27, 1983.

SOUZA, J. C. Variabilidade genética e sistema de cruzamento em populações naturais de umbuzeiro (Spondias tuberosa Arr. Cam.) em Juazeiro. 2000. 86f. Tese (Doutorado em Genética e Melhoramento) Viçosa, MG: Universidade Federal de Viçosa, 2000.

SPITZE, K. Population structure in Daphnia obtusa: quantitative genetic and allozymic variation. Genetics, v.135, p.367-374, 1993.

SUN, A. Q. et al. Isolation and characterization of human glucose-6-phosphate isomerase isoforms containing two different size subunits. Archives of Biochemistry and Biophysics, v.283, n.1, p.120-129, 1990.

TEIXEIRA, M. L.; SOARES, A. R.; SCOLFORO, J. R. S. Variação do teor de tanino da casca de barbatimão [Stryphnodendron adstringens (Mart.) Coville] em 10 locais de Minas Gerais. Ciências e Prática, v.14, n.2, p.229-232, 1990.

TELLES, M. P. C. et al. Caracterização genética de populações naturais de araticunzeiro (Annona crassiflora Mart. - Annonaceae) no Estado de Goiás. Revista Brasileira de Botânica, v.26, n.1, p.123-129, 2003a.

TELLES, M. P. C. et al. Genetic diversity and population structure of Eugenia dysenterica DC. (“cagaiteira”-Myrtaceae) in central Brazil: spatial analysis and implication for conservation and management. Conservation Genetics, v.4, p.685-695, 2003b.
TEKAMP-OLSON, P.; NAJARIAN, R.; BURKE, R. $\mathrm{L}$. The isolation, characterization and nucleotide sequence of the phosphoglucoisomerase gene of Saccharomyces cerevisiae. Gene, v.73, n.1, p.153-161, 1988.

TIHANYI, K. et al. Purification and characterization of alcohol dehydrogenase from soybean.

Phytochemistry, v.28, p.1335-1338, 1989.

TONG, W. F.; LIN, S. W. Purification and biochemical properties of rice alcohol dehydrogenase. Botanical Bulletin of Academia Sinica, v.29, p.245-253, 1988.

VIEGAS, M. P. et al. Diversidade genética e tamanho efetivo de duas populações de Myracrodruon urundeuva Fr. All., sob conservação ex situ. Revista Árvore, v.35, n.4, p.769-779, 2011.

WATANABE, L. et al. Purification, crystallization and preliminary X-ray diffraction analysis of royal palm tree (Roystonea regia) Peroxidase. Acta Crystallographica Section F: Structural Biology and Crystallization Communications, v.63, p.780-783, 2007.

WEEDEN, N. F.; WENDEL, J. F. Genetics of plant isozymes. In: SOLTIS, D. E.; SOLTIS, P. S. (Ed.).

Isozymes in plant biology. London: Chapman and Hall, 1990. p.46-72.

WEN, M. et al. Development of EST-SSR and genomicSSR markers to assess genetic diversity in Jatropha Curcas L. BMC Research Notes, v.3, p.42, 2010.

WORKMAN, P. L. The analysis of simple genetic polymorphism. Human Biology, v.41, n.1, p.97-114, 1969.

WRIGHT, S. The general structure of populations. Annals of Eugenics., v.15, p.323-354, 1951.

YAGI, T. et al. Purification and characterization of aspartate aminotransferase isoenzymes from rice bran. Bioscience, Biotechnology, and Biochemistry, v.57, p.2074-2080, 1993. 